\title{
Impact of patient motivation on compliance and outcomes for incontinence
}

\author{
Phil Reed $^{1}$, Lisa A. Osborne ${ }^{2}$, C. Mair Whittall ${ }^{2}$, and Simon Emery ${ }^{2}$ \\ ${ }^{1}$ Swansea University, UK; ${ }^{2}$ Swansea Bay University Health Board, UK.
}

\author{
Correspondence address: Professor Phil Reed, \\ Department of Psychology, \\ Swansea University, \\ Singleton Park, \\ Swansea, SA2 8PP, UK. \\ Tel.: 01792602047. \\ e-mail: p.reed@swansea.ac.uk
}

Conflicts of Interest: There are none.

Short title: Patient motivation and physiotherapy outcomes. 


\begin{abstract}
Objectives: Physiotherapy treatment for pelvic floor dysfunction in women produces variable outcomes, and discovering predictors of outcome is important for developing service provision and treatment planning. The study aimed to determine whether patient motivation impacts treatment compliance and outcome for physiotherapy treatment in a cohort of patients with pelvic floor dysfunction.
\end{abstract}

Design, setting and participants: A prospective observational study of 141 consecutively referred adult female patients to the physiotherapy clinic of an urogynaecological outpatients unit at a UK hospital.

Intervention: A 6-month (one group session per month) physiotherapy pelvic floor muscle training programme, which included a home-exercise programme.

Main outcome measures: Oxford Grading System and Queensland Pelvic Floor Questionnaire ratings of pelvic floor function were taken pre- and post-intervention, and the University of Rhode Island Change Assessment Scale assessed patient motivation-to-change pre-intervention.

Results: Motivation-to-change predicted attendance at the intervention sessions, and also patient-rated pelvic symptoms improvements in function. Patients with higher baseline motivation-to-change also reported greater patient-rated pelvic symptoms improvements, given the same clinician-rated muscle strength improvement in pelvic floor function.

Conclusions: Patient motivation impacts physiotherapy treatment adherence and outcomes, and should be considered as part of future assessment/screening procedures.

Clinical Trial Registration: NCT02549157.

Keywords: motivation-to-change; clinician-reported outcome measure; patientreported outcome measure; attendance; pelvic floor dysfunction; PFMT. 
Physiotherapy treatment for pelvic floor dysfunction in women can be effective $e^{1,2}$ and is also safe, acceptable, and cost-efficient ${ }^{3}$. However, a consideration that prevents more widespread usage of physiotherapy treatment for this problem is that its outcomes can be variable $^{4}$, and identifying the predictors of its success is a key objective for treatment development and service planning. To date, there have been few studies that have explored these predictors. It is known that physical dysfunction is not necessarily a primary predictor of physiotherapy outcome in this context ${ }^{5,6}$. This stands in contrast to the role of psychological variables (e.g., depression, anxiety) that do appear to play a role in the positive prognosis for physiotherapy for pelvic floor dysfunction ${ }^{7,8}$. However, the full range of psychological predictors of such treatment outcome is far from established, as there have been very few such investigations.

The goal of the present investigation is to develop this under-researched area, and to explore whether a psychological variable - the patient's motivation-to-change ${ }^{9}$ - which known to impact treatment outcomes in other areas, is implicated in the prognosis for physiotherapy treatment for pelvic floor dysfunction. Physiotherapy treatment for pelvic floor dysfunction often takes the form of Pelvic Floor Muscle Training (PFMT) programmes. These PFMT programmes require active patient participation to achieve their impact including attendance at classes, and often behaviour change to affect the desired outcomes. As patient participation (co-production) is central to the goals of the treatment, then the outcomes may well be subject to influence from the psychological characteristics of the patient $^{7,8}$. Given these considerations, the patient's motivation-to-change seems to be a strong candidate for a psychological predictor of outcome for pelvic floor physiotherapy treatment ${ }^{8}$.

The widely adopted Trans-theoretical Model (TTM) of intentional behaviour change ${ }^{9}$, suggests that an individual progresses through a number of 'motivational stages' before 
behaviour change is achieved. According to this model, there are four stages of the change process for an individual: Precontemplation is characterised by only a limited awareness of the problem, or, if the individual knows about the problem, a lack of intention to change; Contemplation involves an awareness of the problem, and often a consideration of the impact of that problem, but without any intention to act on these considerations; the Action stage comprises the individual modifying their behaviour as intended, often utilising aspects learned during the 'contemplation' stage; and the Maintenance stage involves a prolonged engagement with the behaviours needed to maintain any gains and/or avoid a return of the problem.

This model has been shown to have strong predictive validity in many health settings, such as those involving treatment for alcohol abuse ${ }^{10,11}$, illicit drug use $\mathrm{e}^{12-15}$, cigarette smoking $^{16-18}$, criminal offending ${ }^{19}$, and excessive gambling ${ }^{20}$. However, no work has been conducted in terms of exploring how such motivation-to-change may be used as a predictive tool for physiotherapy treatment for pelvic floor dysfunction. It may be that patients who are not yet ready and motivated to commit to long-term physiotherapy treatment will not benefit from that intervention, and discovery of such patient predictors of outcome would be a step toward better matching treatment to patients at a point when they will most benefit from that treatment.

To this end, the current study employed a prospective cohort design to assess the impact of patient motivation at intake on their compliance (as assessed by attendance at sessions) with a physiotherapy treatment for pelvic floor dysfunction. In addition, it also examined the relationship between initial motivation-to-change and the outcomes of the intervention, as assessed both by a clinician-reported outcome measure (CROM) and a patient-reported outcome measure (PROM). The use of both CROM and PROM of pelvic floor function is important, as, irrespective of the change in function from the clinician's 
perspective, the patient's perception of that change is critical in assessing the outcome of the intervention $^{21}$. It may well be that there is no necessary relationship between the two ${ }^{22}$; a finding that has been reported in other conditions ${ }^{23}$. The use of the motivation-to-change scale allows investigation of another issue in the context of the impact of this patient variable on PFMT outcomes. If a patient has higher motivation-to-change, then it has been suggested that they will tend to perceive smaller changes in clinician-rated outcomes more positively than those lacking in this motivation ${ }^{24}$. Theoretically, the presence of a motivational state in behavioural psychology is often termed an establishing operation for sensitivity to environmental changes connected to that state ${ }^{25}$. Given this, it might be expected that there may be differences in pelvic floor outcomes when measured by clinician-rated and patientrated assessments, with patients expressing higher motivation-to-change reporting greater patient-rated benefits from smaller clinician-rated changes.

In summary, the study aimed to determine whether patient motivation impacts treatment compliance and outcome for physiotherapy treatment, and whether it impacts patient perception of clinician-rated changes in muscle strength, in a cohort of patients with pelvic floor dysfunction.

\section{Method}

\section{Participants}

Of 202 consecutively-referred adult female patients to the urogynaecological physiotherapy outpatients' service at an NHS teaching hospital, who were invited to participate, 141 patients agreed and completed the questionnaires. The mean age and standard deviation of the participants was $52(\mathrm{SD}=13$, range $=21-86)$ years. Participants were referred with either a single or combination of symptoms: 22/141 (16\%) stress incontinence; 7/141 (5\%) urge incontinence; 55/141 (39\%) mixed incontinence; 4/141 (3\%) 
faecal incontinence; 21/141 (15\%) prolapse alone; and 32/141 (23\%) prolapse with incontinence. Patients referred for third and fourth degree perineal tears, post-operative rehabilitation, or urogynaecological indications were excluded from the study. The mean BMI of the patients was 30.02 (SD 6.96; range $=18.00-62.90)$.

\section{Materials}

Modified Oxford Grading ${ }^{26}$ is a validated clinician-rated assessment that quantifies the strength of pelvic floor contraction. Patients are scored from $0-5$, where 0 is no pelvic floor contraction and 5 is very strong: elevation of examiner's finger against strong resistance $e^{27,28}$.

Queensland Pelvic Floor Questionnaire ${ }^{29}$ is a validated patient-rated female pelvic floor questionnaire. It has sections related to bladder dysfunction, bowel dysfunction, prolapse, and sexual dysfunction. Each of the subscales produces a score from 0 to 10 , and the sum of the scores gives an overall pelvic floor dysfunction (range $0-40$ ). Greater scores represent worse function. The internal reliability (Cronbach $\alpha$ ) of the scales range between 0.72 and $0.95^{30}$. Analyses of the item responses to the overall scale for the present sample produced an internal reliability $(\alpha)$ of 0.81 .

University of Rhode Island Change Assessment Scale (URICA) ${ }^{31}$ is a commonly used self-assessment designed to measure an individual's motivation-to-change their behaviour. It consists of 32 statements that participants endorse on a 5-point scale (strongly agree to strongly disagree). The items load on four scales, including: Precontemplation, Contemplation, Action, and Maintenance, and each scale gives a score between 0 (low on that dimension) and 7 (high on that dimension). The sum of the scores for Contemplation, Action, and Maintenance minus Precontemplation gives a Readiness-to-change score. The internal reliability $(\alpha)$ for the scales has been reported to range between .88 and $.89^{26}$. 
Analyses of the responses to the items in the scales for the present sample produced internal reliabilities $(\alpha)$ of: Precontemplation $=.81$; Contemplation $=.94 ;$ Action $=.92$; and Maintenance $=.86$, with the overall $\alpha=.92$.

\section{Intervention}

The physiotherapy treatment consisted of 2 individual appointments, and 6 x 60min group sessions (7-8 patients per group), over a 6 month period. The individual appointments were taken by one of the clinical physiotherapists, and were held at the start and end of the PFMT programme. These individual appointments involved vaginal examination to assess vaginal muscles and tissues and pelvic floor strength, in order to assess the patient's pelvic floor exercise technique quality. The PFMT group-based programme provided training in pelvic floor exercises and in identifying and isolating correct muscle groups, as well as educating patients about the anatomy and function of the pelvic floor muscles and the lumbosacral spine region. Group sessions were led by a clinical physiotherapy specialist, a senior physiotherapist in women's health, a surgical nurse specialist, or a psycho-sexual counsellor, as appropriate, who each saw all groups, and were not assigned to one particular group. The sessions also were structured to provide information and enhance awareness regarding: (1) the anatomy and function of the pelvic floor muscles; (2) back and spinal care, as well as posture; (3) medical and surgical management of pelvic floor conditions; (4) psycho-sexual issues; (5) the anatomy of the intestines and bowel, and colorectal problems; and (6) physiotherapy management of Pelvic Floor Dysfunction (PFD) and available aids. In addition, each session provided training in pelvic floor exercises and advice about the behavioural management of continence, such as fluid intake, bladder drill, how to contract pelvic floor muscles before and during increases in abdominal pressure ('the knack'), double voiding, and helpful activities. 
Patients were directed to practice the exercises at home, on a daily basis (mornings and evenings), between the hospital sessions. At the start of the programme, the patients were advised to start with 5 rapid squeezes of their pelvic floor muscles, holding each squeeze between 1-3s, if possible, and then releasing. Patients were encouraged to progressively increase the number and duration of squeezes over the course of the programme, but to primarily focus on the quality of their technique. The goal was to accomplish 10 long squeezes, holding for up to 10s, followed by 10 short squeezes, at least two to three times a day.

\section{Procedure}

On admittance, the participants underwent assessment of their pelvic floor strength by a clinician using the Modified Oxford Grading system. The same clinician rated all patients at both time points. Participants also provided their assessments of their condition using the Queensland Pelvic Floor Questionnaire. They also gave data regarding other demographic characteristics. In addition, the participants also completed a questionnaire to assess their Readiness-to-change (URICA). The participants then progressed through the programme, attending one session every month for the six months. On completion of the programme, the clinician-rated (Modified Oxford Grading) and patient-rated (Queensland Pelvic Floor Questionnaire) assessments of pelvic floor function were repeated.

\section{Analyses Plan}

After assessment of the descriptive statistics, two sets of analyses were planned. A series of stepwise regressions were conducted to determine whether patients' readiness-tochange (sum of Contemplation, Action, and Maintenance, minus the Precontemplation) predicted treatment outcomes: completion of the PFMT programme; change in clinician-rated 
muscle strength (Oxford); and change patient-rated pelvic symptoms (Queensland). In all cases, patient age, patient BMI, clinician-rated intake muscle strength (Oxford), and patientrated intake pelvic symptoms, were entered in step 1, and readiness-to-change was entered in the step 2 to assess its independent contribution. For completion, this was a logistic regression as the outcome is a binary variable (completion versus non-completion), for change in clinician- and patient-rated symptoms, multiple regressions were conducted. In addition, for each of the three outcomes, as the readiness-to-change score does not allow separate assessment of each change scale, multiple regressions were conducted using the four subscales of the URICA as predictors of each outcome. To assess whether readiness-tochange impacted patient perception of symptom improvement, the relationship between change in patient-rated pelvic symptom (Oueensland) and change in clinician-rated muscle strength (Oxford) was assessed for those higher and lower in terms of readiness to change.

\section{Results}

Table 1 about here

Table 1 shows the means (standard deviations) for the clinician-rated (Oxford) and patient-rated (Queensland) assessments of pelvic floor function for the whole sample, as well as separately for the patients who attended all of the sessions in the PFMT programme (completers), and for the patients who did not (non- completers). There were 68/141 (48\%) completers, and 73/141 (52\%) non-completers. Those who completed the PFMT programme had less severe clinician-rated pelvic floor muscle weakness (Oxford) at intake, compared to those who did not complete the programme. However, patient-rated pelvic floor function (Queensland) did not differ between the two groups at intake. For those patients who 
completed the sessions, there was a statistically significant improvement in clinician-rated (Oxford) muscle strength over the course of treatment (intake mean $=2.55$, SD $=.73$; endpoint mean $=3.08, \mathrm{SD}=.0 .83 ; t(67)=4.42, p<.001, d=.61)$. There was also a statistically significant improvement in patient-assessment (Queensland) muscle strength (intake mean = 31.98, $\mathrm{SD}=13.40$; end-point mean $=26.32, \mathrm{SD}=13.79 ; t(67)=2.24, p<.05, d=.29)$.

Table 2 about here

Table 2 shows the means (standard deviations) for the four stages of change scales, Precontemplation, Contemplation, Action, and Maintenance (URICA). It also shows their correlations with the patients’ age, BMI, clinician-rated muscle strength (Oxford), and patient-rated pelvic symptoms (Queensland), at intake. Inspection of these data shows no significant relationships between any of the stages of change scores and patient age, patient BMI, clinician-rated muscle strength (Oxford), or patient-rated pelvic symptoms (Queensland). The only exception was a small positive relationship between contemplation scores and BMI.

\section{Motivation-to-change}

Completion: The addition of readiness-to-change as step 2 of a stepwise logistic regression produced a significant increase in predictive accuracy of completion, $-2 L L=$ $61.62, X^{2}(1)=4.22, p=.040$. Of the individual predictors, readiness-to-change $(\beta=.470, p=$ .050 , odds ratio $=1.597)$, and BMI $(\beta=-.104, p=.029$, odds ratio $=.902)$, were significant independent predictors of completion; but age $(\beta=-.038, p=.160$, odds ratio $=.962)$, clinician-rated intake muscle strength $(\beta=.603, p=.108$, odds ratio $=1.828)$, and patient- 
rated intake pelvic symptoms $(\beta=-.037, p=.142$, odds ratio $=.964)$, were not significantly related to completion.

When the four readiness-to-change subscales were used as predictors of completion, the logistic regression was significant, $-2 L L=185.44, X^{2}(4)=9.66, p=.046$. Of the individual predictors, Action $(\beta=.984, p=.018$, odds ratio $=2.675)$ was a significant independent predictor of completion; but Precontemplation $(\beta=-.171, p>.30$, odds ratio $=$ .843), Contemplation $(\beta=-.394, p>.30$, odds ratio $=.874)$, and Maintenance $(\beta=-.501, p=$ .083 , odds ratio $=.606)$, were not significantly related to completion.

Clinician-rated muscle strength (Oxford): The addition of readiness-to-change as step 2 of a stepwise multiple regression did not produce a significant increase in predictive accuracy of completion, change $R^{2}=.028, F(1,24)=1.68, p=.207$. Of the individual predictors, clinician-rated intake muscle strength $(\beta=-1.038, p<.001)$ was a significant independent predictors of change in clinician-rated muscle strength; but age $(\beta=-.013, p>$ $.30)$, BMI $(\beta=-.018, p>.30)$, patient-rated intake pelvic symptoms $(\beta=.006, p>.30)$, and readiness-to-change $(\beta=.114, p=.207)$, were not significantly related to change in clinicianrated muscle strength.

When the four readiness-to-change subscales were used as predictors of change in clinician-rated muscle strength, the multiple regression was not significant, $R^{2}=.059, F<1$. None of the individual predictors were significantly related to change in clinician-rated muscle strength: Precontemplation, $\beta=-.010, p>$.30; Contemplation, $\beta=.215, p>.30$; Action, $\beta=.178, p>$.30; Maintenance, $\beta=-.275, p=.238$.

Patient-rated pelvic symptoms change: The addition of readiness-to-change as step 2 of a stepwise multiple regression produced a significant increase in predictive accuracy of completion, change $R^{2}=.246, F(1,17)=8.82, p=.032$. Of the individual predictors, readiness-to-change $(\beta=1.137, p=.045)$ was a significant independent predictors of change 
in patient-rated muscle strength; but age $(\beta=.063, p>.30)$, BMI $(\beta=-.269, p>.30)$, clinician-rated intake muscle strength $(\beta=1.232, p>.30)$, and patient-rated intake pelvic symptoms $(\beta=.006, p>.30)$, were not significantly related to change in patient-rated muscle strength.

When the four readiness-to-change subscales were used as predictors of change in patient-rated muscle strength, the multiple regression was not significant, $R^{2}=.135, F(4,45)$ $=1.68, p=1.71$. Of the individual predictors, Action $(\beta=6.803, p=.043)$ was significantly related to change in patient-rated pelvic symptoms; but Precontemplation $(\beta=1.860, p>$ $.30)$, Contemplation $(\beta=-.634, p>.30)$, and Maintenance $(\beta=-1.151, p>.30)$ were not significantly related to change in patient-rated pelvic symptoms.

\section{Perception of Change}

A regression analyses determined the degree to which changes in clinician-rated muscle strength (Oxford) were related to changes in patient-rated pelvic symptoms (Queensland). This regression produced a non-significant model: Queensland change $=-2.15$ -.66(Oxford change), $r^{2}=.007, p>.60$. However, when the sample was split into those whose scores were below or above 11 on the readiness-to-change scale (indicating those at the Precontemplation or Contemplation stages, or those at the Action or Maintenance stages) ${ }^{31}$, the relationship between the clinician-rated muscle strength changes and patientrated pelvic symptoms changes was found to be different in the two groups. For those at a lower stage of readiness-to-change $(n=122)$, the regression model was found to be: Queensland change $=.56-4.35 *($ Oxford change $)$; which was statistically significant, $r^{2}=$ $.304, p<.01$. For those at a higher stage of readiness-to-change $(n=19)$, the regression model was found to be: Queensland change $=-8.00+6.00 *($ Oxford change $)$; which was statistically significant, $r^{2}=.460, p<.05$. The difference in the beta values of the two 
regression models (-4.35 for the lower readiness versus +6.00 for the higher readiness-tochange) suggests that, for any given change in the clinician-rated muscle strength change, a much greater chance of change in the patient-rated pelvic symptoms occurred for the higher motivation group. The difference between the two beta values was found to be statistically significant, $t(137)=4.24, p<.01$.

\section{Discussion}

The current study aimed to determine whether patient motivation-to-change would impact attendance and outcomes in a physiotherapy intervention for pelvic floor dysfunction. The findings were that the PFMT programme produced statistically significant improvements in both clinician-rated muscle strength and patient-rated pelvic symptoms, in line with previous findings ${ }^{1,4,7}$. However, motivation-to-change impacted both attendance at the PFMT sessions, and patient-rated pelvic symptoms. Those with higher levels of readiness-to-change were more likely to attend all of the PFMT sessions, and were more likely to report greater patient-rated pelvic symptom improvements. This was particularly true for those at the Action stage of change; where the recognition of a problem and the intention to overcome the problem have translated into behaviour ${ }^{9}$. However, there was no such relationship with the clinician-rated muscle strength change in pelvic floor function.

These findings relating motivation-to-change and attendance and outcomes for PFMT are novel for this treatment, but they are similar to those obtained from other patient populations ${ }^{10,32,33}$. Given this, the results suggest that the patient's motivation may be a significant factor in determining the likelihood of that patient benefiting from the PFMT programme. At the very least, if a patient does not have the necessary motivation-to-change, they will simply not attend the classes, and will most likely not show any improvements in 
pelvic floor function. This finding, coupled with previous explorations of the impact of motivational interviewing on PFMT outcomes ${ }^{8}$, imply that more focus should be given to these psychological variables in assigning patients to different treatment regimes for pelvic floor function. In addition, providing adjunct psychological support in addition to the PFMT for patients undergoing such treatment may be beneficial ${ }^{8}$.

Research into the impacts of psychological variables on physiotherapy treatments for pelvic floor dysfunction is at an early stage, but there are a number of studies that have shown the importance of considering these factors ${ }^{5,7,8}$. For example, studies ${ }^{7}$ have demonstrated that higher pre-treatment depression scores are associated with poor outcomes for physiotherapy treatments for pelvic floor dysfunction, and have noted that patient health values also predict compliance and outcomes for this treatment.

An additional finding from the current study was that, when motivation-to-change was higher, patients reported greater patient-rated pelvic symptom improvements for the same improvement change in the clinician-rated muscle strength. This finding was predicted from the theoretical literature relating motivation to perceived outcomes ${ }^{24,25}$, but has not until this current study actually been empirically demonstrated in this (nor in any other) context. Apart from the theoretical interest that this finding produces, it also has some practical implications. If patients' motivation-to-change is enhanced prior to, or even during, $\mathrm{PFMT}^{8}$, then it may be that any clinician-rated muscle strength changes that are brought about by the programme would be perceived more positively by the patients. This should increase patientrated pelvic symptom assessment, and their satisfaction with the treatment. In turn, this may drive a 'virtuous circle' of positive feedback; meaning these patients engage more (better comply) with their PFMT. Moreover, to the extent that patients' satisfaction with the outcome and their self-assessment of their pelvic floor functioning is at least as important as any clinician-rated muscle strength changes in this functioning, this would be a notable 
achievement. The fact that motivation-to-change has an 'amplifying effect on subject assessments of improvement, might go some way towards explaining why this former variable was somewhat more strongly related to patient-rated pelvic symptoms than clinicianrated muscle strength change scores. Of course, patients with higher motivation-to-change may also subjectively feel more negatively towards any reduction in functioning that may experience.

The concept of patient motivation-to-change is relatively new in a medical context, although its importance in other health-related fields is recognised. Clearly, measuring this construct will need further development, and finding appropriate questionnaires is still an issue to be considered. The current URICA has an advantage in that it shows strong validity in a variety of contexts, however, anecdotal reports from the participants suggested that they found the questionnaire too long (32 questions) and somewhat intrusive. This might limit its usefulness, in its full form, in a medical assessment context. It is also important to note that the choice of the other instruments that assess clinician-rated and patient-rated assessments of pelvic health may have influenced the current results. Further studies using a range of different assessment tools would further explore these relationships to extend the validity of the finding, or to point to their limits of generalizability.

In the current study, approximately $50 \%$ of patients attended all of the classes. This figure appears quite low, although a recent survey of PFMT programmes in the UK suggested a mean NHS attendance was just below 75\%, but with a very large variance (attendance at private health centres was approximately $85 \%)^{34}$. Of course, this nonattendance figure includes those who may have attended for some of the 6 classes in the programme, but not all, so is a conservative estimate of attendance. Nevertheless, finding ways to enhance this figure, which may include increasing motivation-to-change, would have potentially strong benefits for patients and services. This is thrown into sharper contrast by considering that 
only 19/141 patients had a high readiness-to-change score, compared to 122/141 patients with a low readiness-to-change score. This may reflect the sample chosen, but is probably reflective of patients referred to public health services in general.

A limitation to the current study was that adherence to the home exercise programme (HEP) was not directly measured. As the HEP was part of the intervention, data on adherence would be useful in future studies to add extra information. It may be that HEP is a different form of PFMT, with different motivational factors associated with it compared to class attendance. There are a number of ways in which this could be measured, including a range of available apps - although all suffer from the problems of self-report.

In summary, the current research is amongst the first that has identified that patient motivation may play a role in treatment compliance and outcomes. These findings suggest that a fuller assessment of the psychological functioning of patients would facilitate treatment regimes in the field of physiotherapy and pelvic floor functioning. In addition to this, motivation assessment may play a role in future screening by providing practitioners the ability to predict the relative likelihood of patients completing their physiotherapy programme, and providing better treatment provisions for the patient, allowing customised PFMT treatment based on psychological status. 
Ethical Approval: Ethical approval was granted to this study by the NRES Committee Region - East Midlands, UK. The trial is registered on clinicaltrials.gov (NCT02549157).

Funding: Funding was provided by the Abertawe Bro Morgannwg University Health Board Research and Development Fund.

Conflicts of Interest: There are none. 


\section{Contribution of the Paper}

- The study determined whether patient motivation impacts treatment compliance and outcome for physiotherapy treatment in a cohort of patients with pelvic floor dysfunction.

- Patients with higher baseline motivation-to-change also reported greater PROM pelvic symptoms improvements, given the same CROM muscle strength improvement in pelvic floor function.

- Patient motivation impacts physiotherapy treatment adherence and outcomes, and should be considered as part of future assessment/screening procedures. 


\section{References}

1. Hay-Smith J, Bo K, Berghmans B, Hendriks E, de Bie R, van Waalwijk van Doorn E. Pelvic floor muscle training for urinary incontinence in women. The Cochrane Library 2002.

2. Bols E et al. Rectal balloon training as add-on therapy to pelvic floor muscle training in adults with fecal incontinence: A randomized controlled trial." Neurourology and Urodynamics 2012; 31: 132-8.

3. National Institute for Health and Clinical Excellence. Urinary Incontinence: The Management of Urinary Incontinence in Women. NICE Clinical Guideline 402006 London: NICE.

4. Hay-Smith EJ, Dumoulin C. Pelvic floor muscle training versus no treatment, or inactive control treatments, for urinary incontinence in women. Cochrane Database Syst Rev 2006; 1: CD005654.

5. Dumoulin C, Bourbonnais D, Morin M, Gravel D, Lemieux MC. Predictors of success for physiotherapy treatment in women with persistent postpartum stress urinary incontinence. Archives of Physical Medicine and Rehabilitation 2010; 91: 1059-1063.

6. Goode PS et al.. Population based study of incidence and predictors of urinary incontinence in black and white older adults. The Journal of Urology 2008; 179: 1449-54.

7. Khan ZA, Whittal CM, Mansell S, Osborne LA, Reed P, Emery S. Effect of depression and anxiety on the success of pelvic floor muscle training for pelvic floor dysfunction. Journal of Obstetrics and Gynaecology 2013.

8. Osborne LA, Whittall CM, Edwards DJ, Emanuel R, Emery S, Reed P. Randomised control trial of a values-based motivational interview support to promote attendance at pelvic floor muscle training physiotherapy treatment. Pelvic, Obstetric, \& Gynaecological Physiotherapy Journal 2016. 
9. Prochaska, J. O., \& DiClemente, C. C. (1982). Transtheoretical therapy: Toward a more integrative model of change. Psychotherapy: Theory, Research \& Practice, 19, 276-288.

10. Carbonari, J. P., \& DiClemente, C. C. (2000). Using transtheoretical model profiles to differentiate levels of alcohol abstinence success. Journal of Consulting and Clinical Psychology, 68, 810-817.

11. Project MATCH research group. (1997). Matching alcoholism treatments to client heterogeneity: Project MATCH post-treatment drinking outcomes. Journal of Studies on Alcohol, 58, 7-29.

12. Belding, M. A., Iguchi, M. Y., \& Lamb, R. J. (1996). Stages of change in methadone maintenance: Assessing the convergent validity of two measures. Psychology of Addictive Behaviors, 10, 157-166.

13. Dunn, C., DeRoo, L., \& Rivara, F. P. (2001). The use of brief interventions adapted from motivational interviewing across behavioral domains: A systematic review. Addiction, 96, $1725-1742$.

14. Pantalon, M. V., Nich, C., Frankforter, T., \& Carroll, K. M. (2002). The URICA as a measure of motivation to change among treatment-seeking individuals with concurrent alcohol and cocaine problems. Psychology of Addictive Behaviors, 16, 299-307.

15. Valesquez, M. M., Carbonari, J. P., \& DiClemente, C. C. (1999). Psychiatric severity and behavior change in alcoholism: The relation of the trans-theoretical model variables to psychiatric distress in dually diagnosed patients. Addictive Behaviors, 24, 481-496.

16. McCusker, J., Bigelow, C., Frost, R., Hindin, R., Vickers-Lahti, M., \& Zorn, M. (1994). The relationships of HIV status and HIV risky behavior with readiness for treatment. Drug and Alcohol Dependence, 34, 129-138. 
17. Prochaska, J. O., \& DiClemente, C. C. (1985). Common processes of change in smoking, weight control and psychological distress. In S. Shiffman \& T. A. Wills (Eds.), Coping and substance abuse (pp. 345-363). New York: Academic Press.

18. Prochaska, J. O., DiClemente, C. C., \& Norcross, J. C. (1992). In search of how people change: Applications to addictive behaviors. American Psychologist, 47, 1102-1114.

19. El-Bassel, N., Schilling, R. F., Ivanoff, A., Chen, D., Hanson, M., \& Bidassie, B. (1998). Stages of change profiles among incarcerated drug-using women. Addictive Behaviors, 23, 389-394.

20. Neighbors, C., Lostutter, T.-W., Larimer, M. E., \& Takushi, R. Y. (2002). Measuring gambling outcomes among college students. Journal of Gambling Studies, 18, 339-360.

21. Bahl JS, Dollman J, Davison K. The development of a subjective assessment framework for individuals presenting for clinical exercise services: A Delphi study. Journal of Science and Medicine in Sport 2016 Feb 5.

22. Amaro JL, Gameiro MO, Padovani CR. Treatment of urinary stress incontinence by intravaginal electrical stimulation and pelvic floor physiotherapy. International Urogynecology Journal 2003;14(3):204-8.

23. Reed P, Osborne LA. The role of parenting stress in discrepancies between parent and teacher ratings of behavior problems in young children with autism spectrum disorder. Journal of Autism and Developmental Disorders 2013;43(2):471-7.

24. Wilson DK, Purdon SE, Wallston KA. Compliance to health recommendations: A theoretical overview of message framing. Health Education Research 1988;3(2):161-71.

25. Mason SA. Establishing Operations. In Encyclopedia of Autism Spectrum Disorders 2013 (pp. 1165-1167). Springer New York.

26. Sapsford R, B.-S. J., Markwell S Women's Health 1998 
27. Brink, CA, Wells TJ, Sampselle CM, Taillie ER Mayer R. A digital test for pelvic muscle strength in women with urinary incontinence. Nurs Res 1994; 43: 352-6.

28. Sampselle CM, Brink CA, Wells TJ. Digital measurement of pelvic muscle strength in childbearing women. Nurs Res 1989; 38: 134-8.

29. Baessler K, O'Neill SM, Maher CF, Battistutta D. An interviewer-administered validated female pelvic floor questionnaire for community-based research. Menopause 2008; 15: 973-7.

30. Baessler K, O’Neill SM, Maher CF, Battistutta D. Australian pelvic floor questionnaire: a validated interviewer-administered pelvic floor questionnaire for routine clinic and research. International Urogynecology Journal 2009;20(2):149-58.

31. McConnaughy, E. A., Prochaska, J. O., \& Velicer, W. F. (1983). Stages of change in psychotherapy: Measurement and sample profiles. Psychotherapy: Theory, Research \& Practice, 20, 368-375.

32. Edens, J. F., \& Willoughby, F. W. (2000). Motivational patterns of alcohol dependent patients: A replication. Psychology of Addictive Behaviors, 14(4), 397.

33. Henderson, M. J., Saules, K. K., \& Galen, L. W. (2004). The Predictive Validity of the University of Rhode Island Change Assessment Questionnaire in a Heroin-Addicted Polysubstance Abuse Sample. Psychology of Addictive Behaviors, 2004, Vol. 18, No. 2, $106-112$.

34. Reed, P., Mann, K., \& Osborne, L.A. (2020). Pelvic Floor Muscle Training services across the UK: A benchmarking survey of POGP members. Journal of Pelvic, Obstetric, and Gynaecological Physiotherapy, 126, 49-57. 
Table 1: Means (and standard deviations) for the clinician-rated muscle strength (Oxford) and patient-rated pelvic symptoms (Queensland) assessments of pelvic floor function for the whole sample, completers, and non-completers, at the intake point.

\begin{tabular}{|c|c|c|c|c|c|}
\hline & $\begin{array}{l}\text { Cohort } \\
(\mathrm{n}=114)\end{array}$ & $\begin{array}{l}\text { Non-completers } \\
(\mathrm{n}=73)\end{array}$ & $\begin{array}{l}\text { Completers } \\
\qquad(\mathrm{n}=68)\end{array}$ & $t$ & $d$ \\
\hline Oxford & $2.38 \quad(0.88)$ & 2.11 & $2.55(0.73)$ & $2.33^{*}$ & .50 \\
\hline Queensland & 33.31 (15.67) & 34.23 (14.97) & $31.98(13.40)$ & $<1$ & .07 \\
\hline
\end{tabular}


Table 2: Means (standard deviations) for the stages of change (URICA) scales, along with their correlations with the patients' age, BMI, clinician-rated muscle strength (Oxford), and patient-rated pelvic symptoms (Queensland) at intake.

\begin{tabular}{|c|c|c|c|c|c|}
\hline & Mean (SD) & Age & $\mathrm{BMI}$ & Oxford & Queensland \\
\hline Precontemplation & $2.23(0.80)$ & .067 & -.077 & -.029 & -.030 \\
\hline Contemplation & $3.86(0.81)$ & -.056 & $.210 *$ & .052 & .126 \\
\hline Action & 3.83 (0.73) & -.082 & .162 & .066 & -.077 \\
\hline Maintenance & $3.25(0.83)$ & .096 & .178 & -.077 & .068 \\
\hline Readiness & $8.70(2.12)$ & -.036 & $.243^{*}$ & -.006 & .109 \\
\hline
\end{tabular}

$* \quad p<.05 ; * * p<.01 ; * * * p<.001$ 